\title{
Influence of accretion and core formation on the light element compositions of planetary cores
}

\author{
REBECCA A. FISCHER ${ }^{1}$ \\ ${ }^{1}$ Harvard University, Dept. of Earth and Planetary Sciences
}

Light elements such as $\mathrm{O}, \mathrm{Si}, \mathrm{S}, \mathrm{C}$, and $\mathrm{H}$ [e.g., 1] in terrestrial planetary cores have a number of important effects, including depressing the melting point of pure iron and driving compositional convection in the Earth's outer core [e.g., 2]. Better knowledge of the light element composition of a planet's core informs our understanding of its thermal structure, geodynamics, and volatile abundance. The core light element budget is largely set during accretion and core formation, so studies into these processes can better constrain core composition.

We have performed a suite of $100 \mathrm{~N}$-body simulations of terrestrial planet accretion in our Solar System [3], which provide plausible mass evolution histories for the planets as well as information about the provenance of their building blocks and its temporal evolution. We have also performed metal-silicate partitioning experiments in the laser-heated diamond anvil cell, which provide insight into the behaviors of Si, O, and C during Earth's core formation [4-5]. Si and O become more siderophile at higher pressures and temperatures, while $\mathrm{C}$ becomes less siderophile.

$\mathrm{N}$-body simulations and experimental partitioning data can be combined in a model of core formation to predict the composition of Earth's core [6]. We find a most likely core composition for Earth of $1.9 \mathrm{wt} \% \mathrm{O}, 5.5 \mathrm{wt} \% \mathrm{Si}$, and $<0.2$ $\mathrm{wt} \% \mathrm{C}$ for model parameters that match the mantle's composition. The $\mathrm{Si} / \mathrm{O}$ ratio of Earth's core is a function of the oxidation state of what it accreted. We have also investigated the composition of the Martian core using a similar model, finding $18.4 \mathrm{wt} \% \mathrm{~S}, 0.1 \mathrm{wt} \% \mathrm{O}$, and negligible Si [7].

[1] Poirier J.-P. (1994) PEPI 85, 319-337. [2] Stevenson D.J. (1981) Science 214, 611-619. [3] Fischer R.A., Ciesla F.J. (2014) EPSL 392, 28-38. [4] Fischer R.A. et al. (2015) GCA 167, 177-194. [5] Fischer R.A. et al. (submitted). [6] Fischer R.A., Campbell A.J., Ciesla F.J. (2017) EPSL 458, 252-262. [7] Brennan M.C., Fischer R.A., Irving J.C.E. (2020) EPSL 530, 115923. 